\title{
A Database of Polarized K3 Surfaces
}

\author{
Gavin Brown
}

\section{CONTENTS}

1. Introduction

2. Families of K3 Surfaces

3. Numerical Unprojection and Weights

4. Using the K3 Database

Acknowledgments

References

2000 AMS Subject Classification: Primary 14J28;

Secondary 14J10, 14Q10

Keywords: K3 surface, elliptic fibration, graded ring, unprojection
We describe a computer-based database of polarized K3 surfaces and explain the meaning of the information it contains. In a precise sense, the database includes all K3 surfaces.

\section{INTRODUCTION}

Many authors have compiled lists of K3 surfaces embedded in weighted projective space (wps). The first of these lists contains the "famous 95" weighted hypersurfaces of Reid [Reid 80, Theorem 4.5] and others. Next there are 84 families of K3 surfaces in codimension 2 computed by Iano-Fletcher [Iano-Fletcher 00, Section 13.8], followed by 70 families in codimension 3 and 142 families in codimension 4, both computed by Altınok [Altınok 98]. Such lists could be continued indefinitely in increasing codimension, since there are countably many deformation families of polarized K3 surfaces, although the construction of explicit equations becomes difficult.

We extend the classification of polarized K3 surfaces to give a list that contains the numerical data of all polarized K3 surfaces in the precise sense of Theorem 2.7 below. Although the list of families of polarized K3 surfaces is infinite, the numerical data we work with behave in a regular way after the first 15,000 or so families are obtained, and so a finite list can summarize the whole classification.

Even so, it is far too large to be reproduced in the way that the existing lists have been. In fact, both the analysis used to create the list and methods of interrogating it are handled by a computer. The resulting list of 24,099 numerical K3 candidates (see Definition 2.5) is known as the K3 database. It was created using the computer algebra system MAGma [Cannon 05, Bosma et al. 97], and it is accessible in three ways: one can run MAGMA itself, connect to the Web interface at [Brown et al. 04] (which runs MAGMA in the background), or install an SQL-style database [Brown and Kerber 05] prepared from the online version. These are discussed in Section 4. Although computer access is the only serious way to address such a 
database, K3 surfaces in low codimension are also available at [Brown et al. 04] including a new list of $163 \mathrm{~K} 3$ surfaces in codimension 5 .

The main results of this paper explain the meaning of the K3 database. We make this explicit in Meanings 2.6, $2.8,2.9,3.6$. Theorem 2.7 explains the sense in which it is comprehensive and the way in which we regard the K3 database as an upper bound for the numerical data of polarized K3 surfaces. An immediate corollary is a sharp lower bound on the degree of polarized K3 surfaces; see Section 4 for MAGMA code that performs this calculation.

Computation 1.1. If $X, A$ is a polarized $\mathrm{K} 3$ surface, then the degree $A^{2}$ of $X$ is at least $1 / 330$. In more detail, both the degree $A^{2}$ and the Picard number $\rho_{X} \leq 20$ of $X$ are bounded below according to the genus $g=h^{0}(X, A)-1$ as follows:

\begin{tabular}{r|cccc}
$g$ & -1 & 0 & 1 & $\geq 2$ \\
\cline { 2 - 5 } lower bound for $A^{2}$ & $1 / 330$ & $1 / 42$ & $1 / 2$ & $2 g-2$ \\
lower bound for $\rho_{X}$ & 10 & 6 & 2 & 1
\end{tabular}

We clear up two points of confusion at once. First, there is no claim that every numerical candidate in the K3 database comes from a polarized K3 surface. Indeed, in Theorem 2.11 we show that one particular candidate does not arise as a K3 surface, at least not in an easy way. Second, while each candidate in the database is given a plausible description as a K3 surface embedded in wps, there is no claim that this description can be realized, even when there is a K3 surface whose invariants match those of the candidate.

More positively, there are various ways in which a candidate in the database may be justified. One is to write down equations for a K3 surface in wps and confirm its properties. This is done for all candidates in codimensions 1, 2, and 3 in [Iano-Fletcher 00] and [Altinok 98]. Another is by unprojection, which is discussed in Section 3 ; this is a "bottom-up" approach, constructing complicated surfaces from easy ones. The reason for discussing it here is not to propose to carry out the unprojection calculations but to explain the descriptions of candidates in the database.

For the rest of this introduction, we explain the purpose of this classification and relate it to others in the literature.

There are many reasons for assembling reasonably large databases of varieties rather than restricting attention to those instances of classification that result in short lists. Belcastro [Belcastro 02], for instance, uses the famous 95 as a testing ground for K3 mirror symmetry.
Johnson and Kollár [Johnson and Kollár 01] construct and use lists of weighted hypersurfaces to find varieties admitting a Kähler-Einstein metric. Corti, Pukhlikov, and Reid [Corti et al. 00] use the famous 95 as the starting point for a systematic and explicit study of birational rigidity and the Sarkisov program for Fano 3-folds. In some of these cases, one could regard lists of varieties as being merely a convenient source of many examples, rather than an essential ingredient. But already Belcastro is hampered by restricting to hypersurfaces, since, not surprisingly, in seeking mirror pairs she finds hypersurfaces whose partner, if it exists, is not another hypersurface in wps.

The main reason for extending the lists as we do is as part of the classification of Fano 3-folds. We explain this briefly; see [Altınok et al. 02] for much greater detail. A 3 -fold $X$ is a Fano 3 -fold if it has at worst $\mathbb{Q}$-factorial terminal singularities and $-K_{X}$ is ample. It is common to insist that moreover, $\operatorname{Pic}(X)=\mathbb{Z}$ and that $-K_{X}$ be a generator. By [Kawamata 92], there are only finitely many deformation families of Fano 3-folds. If the linear system $\left|-K_{X}\right|$ contains an irreducible surface $S$ with only Du Val singularities, then $S$ is a K3 surface and it is polarized by the trace of $-K_{X}$. Such a surface $S$ is called a $K 3$ elephant for $X$, and the vast majority of known Fano 3-folds have a K3 elephant.

The main point of [Altınok et al. 02] is to attempt the converse operation: given a polarized K3 surface $S, A$, construct a Fano 3 -fold $X$ having $S$ as its K3 elephant. This can be regarded as a deformation-extension problem, in which one must include a new variable in the equations of $S$ while maintaining the irreducibility (at the very least) of the locus they define. From this point of view, the K3 database contains a coarse classification of Fano-with-elephant 3-folds as a finite sublist (although exactly which sublist is the interesting point).

There are many other lists of varieties we could mention. Following classifications by Miranda and Persson [Miranda and Persson 89] and others, Shimada and Zhang [Shimada and Zhang 01, Shimada 00] classify K3 surfaces that arise as elliptic fibrations. Kreuzer and Skarke [Kreuzer and Skarke 98] classify K3 surfaces that arise as toric hypersurfaces, and in higher dimension, they classify Calabi-Yau 3-fold toric hypersurfaces [Kreuzer and Skarke 00]. Their famous Calabi-Yau database contains nearly 500 million families of CalabiYau 3-folds; it is not known whether there are infinitely many families. Buckley and Szendrői [Buckley and Szendrői 05, Buckley 03] construct Calabi-Yau 3-folds by methods similar to those we use here, although their in- 
terest is not in constructing lists of varieties but rather in finding examples not already in the vast Kreuzer-Skarke list. More recently, Caravantes [Caravantes 05] computed examples of Fano 3-folds that are quotients of other Fano 3-folds - so-called Fano-Enriques 3-folds - in codimension at most 3, and Kasprzyk [Kasprzyk 06, Kasprzyk 05] computes the classifications of toric Fano 3-folds under various hypotheses.

\section{FAMILIES OF K3 SURFACES}

The methods used here follow Altinok's approach using Hilbert series, as explained in [Altınok et al. 02].

\subsection{Polarized K3 surfaces}

A polarized $K 3$ surface is a pair $X, A$, where $X$ is a surface having only Du Val singularities, trivial canonical divisor $K_{X}=0$, and irregularity $q=0$, while $A$ is an ample divisor on $X$. Recall that a $D u$ Val singularity (or Kleinian or ADE singularity) is the germ at the origin of $\mathbb{C}^{2} / G$, where $G \subset \mathrm{SL}(2, \mathbb{C})$ is a finite group; equivalent conditions, see [Durfee 79] or [Reid 80], include being defined by an equation from the list of ADE normal forms, or imposing no conditions on adjunction so that the canonical class pulls back to a minimal resolution. Throughout this paper, the term "K3 surface" refers to such a pair $X, A$.

2.1.1 Graded Ring of a K3 Surface. A polarized K3 surface has a graded $\operatorname{ring} R(X, A)=\oplus_{n \geq 0} H^{0}(X, n A)$, and the Hilbert series $P_{X}(t)$ of $X, A$ is defined to be the Hilbert series of this graded ring:

$$
P_{X}(t)=\sum_{t \geq 0} h^{0}(X, n A) t^{n} .
$$

Since $A$ is ample, $R(X, A)$ is a finitely generated $k$ algebra and the Proj correspondence embeds $X$ in wps:

$$
X=\operatorname{Proj} R(X, A) \subset \mathbb{P}^{N} \text { for some } \mathbb{P}^{N}=\mathbb{P}\left(a_{0}, \ldots, a_{N}\right),
$$

where we suppose that $R(X, A)$ is minimally generated as a $k$-algebra by homogeneous elements $x_{0}, \ldots, x_{N} \in$ $R(X, A)$ with $\operatorname{deg} x_{i}=a_{i}$. A minimal free resolution of $R(X, A)$ as a $k\left[\mathbb{P}^{N}\right]$-module then exhibits a preferred rational form of the formal power series $P_{X}(t)$ :

$$
P_{X}(t)=\frac{H_{X}(t)}{\prod\left(1-t^{a_{i}}\right)},
$$

where $H_{X}(t)$ is a polynomial, the Hilbert numerator of $X, A$, and the denominator product is taken over the weights $a_{0}, \ldots, a_{N}$ of the wps $\mathbb{P}^{N}$. The codimension of $X, A$ is defined to be the codimension of $X$ in this embedding. The genus of $X, A$ is $h^{0}(X, A)-1$, which is an integer greater than or equal to -1 .
2.1.2 Riemann-Roch and Baskets of Singularities. Altinok's Riemann-Roch formula, Theorem 2.1 below, computes the Hilbert series of a $\mathrm{K} 3$ surface $X, A$. It involves the notion of a basket of quotient singularities, which is explained below, to compute the effect of the singularities of $X, A$ on $h^{0}(X, n A)$.

Theorem 2.1. (Altınok.) [Altınok 98, Theorem 4.6], [Altinok 03, 3.2] Let X, A be a polarized K3 surface. Then

$$
\begin{aligned}
P_{X}(t)= & \frac{1+t}{1-t}+\frac{t(1+t)}{(1-t)^{3}} \frac{A^{2}}{2} \\
& -\sum_{\mathcal{B}} \frac{1}{\left(1-t^{r}\right)} \sum_{i=1}^{r-1} \frac{\overline{b i}(r-\overline{b i}) t^{i}}{2 r},
\end{aligned}
$$

where

$$
A^{2}=2 g-2+\sum_{\mathcal{B}} \frac{b(r-b)}{r} .
$$

In these formulas, $\mathcal{B}$ is a collection of cyclic quotient singularities $\frac{1}{r}(a,-a)$ at which the polarizing divisor $A$ restricts to the eigensheaf $\mathcal{L}_{a}$ of the quotient. The notation $\bar{x}$ denotes the minimal nonnegative residue of $x$ modulo $r$, and $b=\bar{b}$ satisfies $\overline{a b}=1$.

The collection $\mathcal{B}$ of cyclic quotient singularities is called the basket of singularities of $X, A$. It computes the contribution of the actual singularities of $X, A$ to Riemann-Roch. In general the singularities of $X$ may differ from $\mathcal{B}$. This phenomenon has been well known since [Reid 80] and [Reid 87], although here we need to know how baskets arise.

If $p \in X$ is a $\mathrm{Du}$ Val singularity, then it is also polarized by $A$. This global polarizing divisor restricts to some element of the local class group of $p \in X$. Taking a small analytic neighborhood $p \in U \subset X$, there is a deformation of $U, A_{\mid U}$ such that the general fiber $U_{t}, A_{t}$ has only cyclic quotient singularities, and at each such $q \in U_{t}$ the divisor $A_{t}$ restricts to a generator of the local class group. Thus $q \in U_{t}$ is of type $\frac{1}{r}(a,-a)$ for coprime $0<a<r$. Let $\mathcal{B}_{p}$ be the collection of these polarized cyclic quotient singularities. This collection $\mathcal{B}_{p}$ is uniquely determined by the polarized singularity $p \in X$. Finally, $\mathcal{B}$ is the collection of all $\mathcal{B}_{p}$ as $p$ runs through the Du Val singularities of $X$. The following result is implicit in [Reid 87, (9.4)] .

Lemma 2.2. In the notation above, let $\Gamma_{p}$ be the dual graph of the resolution of $p \in X$, and $\Gamma_{q_{1}}, \ldots, \Gamma_{q_{k}}$ those of the basket $\mathcal{B}_{p}$. Then the disjoint union $\cup \Gamma_{q_{i}}$ embeds as a subgraph of $\Gamma_{p}$ in such a way that no two components $\Gamma_{q_{i}}$ and $\Gamma_{q_{j}}$ for $i \neq j$ are joined by an edge of $\Gamma_{p}$. 
Proof: By [Reid 87, (9.4) and (4.10)] (suitably reordered), the only polarized Du Val singularities that lead to a nonempty basket are as follows:

\begin{tabular}{c|c}
$p \in X$ & basket \\
\hline $\mathrm{A}_{n k-1}$ & $k \times A_{n-1}$ \\
$\mathrm{D}_{2 k+1}$ & $A_{3}+(k-1) \times A_{1}$ \\
$\mathrm{D}_{k+2}$ & $2 \times A_{1}$ \\
$\mathrm{E}_{6}$ & $2 \times A_{2}$ \\
$\mathrm{D}_{2 k}$ & $k \times A_{1}$ \\
$\mathrm{E}_{7}$ & $3 \times A_{1}$
\end{tabular}

In each case, the dual graphs of the basket can be arranged as a disconnected subgraph of $\Gamma_{p}$ as claimed.

It would be convenient to know that $X$ deforms to a K3 surface with singularities equal to the basket, but we do not know that or need it.

Proposition 2.3. If $\mathcal{B}$ is a basket for a $K 3$ surface of genus $g$, then

$$
\sum_{\mathcal{B}}(r-1) \leq 19 \quad \text { and } \quad 2 g-2+\sum_{\mathcal{B}} \frac{b(r-b)}{r}>0 .
$$

Furthermore, if the singularities of $\mathcal{B}$ lie on a surface $Y$, then the minimal resolution of these singularities must not contain 17 disjoint-2-curves, and all coefficients of the power series $P(t)$ computed by formula (2-2) are nonnegative.

Proof: If the singularities of $X$ are equal to those of the basket (as polarized singularities), then all the claims are standard: the first comes from the bound on the Picard rank of the resolution; the second is $A^{2}>0$; the third is a standard consequence of the Torelli theorem. Even if the singularities of $X$ are not those of $\mathcal{B}$, the second inequality holds automatically, since the basket computes $A^{2}$ exactly.

In general, Lemma 2.2 shows that the number of exceptional curves in a resolution of $X$ is at least that in the resolution of its basket, and moreover, if one can find $k$ disjoint -2-curves in the resolution of the basket, then the same is true for the resolution of singularities of $X$ itself.

Computation 2.4. Let $B_{g}$ be the set of baskets that appear in Riemann-Roch for a K3 surface with genus $g \geq-1$. Then $B_{g}$ is finite and of size as given in the following table:

$$
\begin{array}{c|cccc}
g & -1 & 0 & 1 & \geq 2 \\
\hline \# B_{g} & 4281 & 6479 & 6627 & 6628
\end{array}
$$

Moreover, $B_{g}=B_{2}$ whenever $g \geq 3$.

When $g \leq 2$, this is the result of a simple computer enumeration of all possible baskets of singularities of type $\frac{1}{r}(a,-a)$ for coprime $0<a<r$ satisfying the four conditions of Proposition 2.3. The fact that $B_{g}=B_{2}$ when $g \geq 3$ is immediate from the form of the degree condition $A^{2}>0$. The "missing" basket in genus 1 is the empty one: there is no nonsingular K3 surface with $g=1$.

\subsection{The Meaning of the K3 Database}

The K3 database is intended to represent all possible $\mathrm{K} 3$ surfaces $X, A$. Here we say in what sense every K3 surface appears in the database, and conversely, we begin to see to what extent items in the database come from K3 surfaces.

Definition 2.5. A numerical $K 3$ candidate is a pair $(g, \mathcal{B})$, where $g \geq-1$ is an integer and $\mathcal{B}$ is a basket from the set $B_{g}$ constructed in Computation 2.4.

A numerical K3 candidate contains exactly the data needed to compute a Hilbert series using the formula of Theorem 2.1.

Meaning 2.6. The K3 database is a finite set $\mathcal{D}_{\mathrm{K} 3}$ whose elements are numerical K3 candidates. It includes the candidates $(g, \mathcal{B})$ for $-1 \leq g \leq 2$ and all $\mathcal{B} \in B_{g}$.

For each candidate $\xi=(g, \mathcal{B})$, we define formally a degree, denoted by $A_{\xi}^{2}$, and a Hilbert series, denoted by $P_{\xi}(t)$, by the formulas $(2-3)$ and $(2-2)$ respectively.

Theorem 2.7. (Completeness of the $\mathbf{K} 3$ database.) Let $X, A$ be a polarized K3 surface of genus $g$. Then $X, A$ is represented in the $K 3$ database $\mathcal{D}_{\mathrm{K} 3}$ as follows:

- If $g \leq 2$, then there is a numerical K3 candidate $\xi=(g, \mathcal{B}) \in \mathcal{D}_{\mathrm{K} 3}$ with

$$
A^{2}=A_{\xi}^{2} \quad \text { and } \quad P_{X}(t)=P_{\xi}(t) .
$$

- If $g \geq 3$, then there is a numerical K3 candidate $\xi=(2, \mathcal{B}) \in \mathcal{D}_{\mathrm{K} 3}$ with

$$
A^{2}=A_{\xi}^{2}+2(g-2)
$$

and

$$
P_{X}(t)=P_{\xi}(t)+\frac{t(1+t)}{(1-t)^{3}}(g-2) .
$$


Proof: If $g \leq 2$, then this follows immediately from Theorem 2.1 and Proposition 2.3. When $g \geq 3$, it holds because $B_{2}=B_{g}$ from Computation 2.4 implies that the formulas $(2-3)$ and (2-2) differ from the $g=2$ case only by the $2 g$ term in $A^{2}$.

2.2.1 Weights and Codimension. The K3 database includes extra information about each entry: each $\xi \in$ $\mathcal{D}_{\mathrm{K} 3}$ has a sequence of weights $\left(a_{0}, \ldots, a_{N}\right)$ associated with it with positive integers $a_{i}$. The hope is that a K3 surface $X, A$ exists with numerical data $\xi$ and embedded by (all multiples of) $A$ in $\mathbb{P}^{N}\left(a_{0}, \ldots, a_{N}\right)$.

The naive method to generate such weights generalizes the first examples such as those in [Altinok et al. 02, Section 1]; variations of it are described in [Iano-Fletcher 00, Section 18]. If $P_{\xi}(t)=1+p_{1} t+p_{2} t^{2}+\cdots$ is the Hilbert series of some ring $R$, then $R$ must have $p_{1}$ generators in degree 1 . We compute $(1-t)^{p_{1}} P_{\xi}=1+p_{k}^{\prime} t^{k}+\cdots$, where $p_{k}^{\prime}$ is the first nontrivial coefficient. If $p_{k}^{\prime}>0$, then $R$ must also have $p_{k}^{\prime}$ generators in degree $k$; in that case we compute $(1-t)^{p_{1}}\left(1-t^{k}\right)^{p_{k}^{\prime}} P_{\xi}$ and continue. If $p_{k}^{\prime}<0$, then $R$ must have at least $\left|p_{k}^{\prime}\right|$ relations in degree $k$; in that case we stop the calculation and let the weights of $\xi$ be the collection of weights of all generators deduced so far.

Thus, just as in Section 2.1, the weights determine a preferred rational expression for the corresponding Hilbert series. Its numerator is again called the Hilbert numerator and is denoted by $H_{\xi}(t)$ in this context. (In principle, it is possible that the calculation breaks down too soon and $H_{\xi}$ is not a polynomial, but in practice this does not happen.) In this way, the weights of $\xi$ determine a prediction of a $\mathrm{K} 3$ surface $X \subset \mathbb{P}^{N}\left(a_{0}, \ldots, a_{N}\right)$ that realizes $\xi$. With this in mind, the codimension of $\xi$ is defined to be $N-2$.

Meaning 2.8. The $\mathrm{K} 3$ database $\mathcal{D}_{\mathrm{K} 3}$ comprises all pairs $(g, \mathcal{B})$ with $g \leq 2$ and $\mathcal{B} \in B_{g}$ together with those pairs with $3 \leq g \leq 9$ having codimension at most 7 . For each genus $g$, the pairs $(g, \mathcal{B})$ are listed in increasing order of Hilbert series.

The order on Hilbert series is of course the natural lexicographic order. The weights, and hence the codimension, are those computed in Section 3 using more systematic methods than the naive one above. The number of numerical K3 candidates per genus and codimension is listed in Table 1.

2.2.2 Degenerations of Graded Rings. Of course, the Hilbert series of a graded ring does not determine that ring. Whichever method is used to compute the weights, they are not expected to match every K3 surface $X, A$ with given Hilbert series.

Meaning 2.9. If $X, A$ is a $\mathrm{K} 3$ surface with genus less than or equal to 2 , then the Hilbert series $P_{X, A}(t)$ of $X$ will be that of some $\xi \in \mathcal{D}_{\mathrm{K} 3}$. However, the weights assigned to the candidate $\xi$ will not necessarily be those of a set of generators of the graded ring $R(X, A)$.

Consider the well-known example of a general complete intersection of equations of degrees 2 and 4 ,

$$
Y_{2,4} \subset \mathbb{P}^{4}(1,1,1,1,2),
$$

where the weight-2 variable does not appear in the degree-2 equation. This K3 surface has the same Hilbert series as the quartic surface in $\mathbb{P}^{3}$. The corresponding $\xi \in \mathcal{D}_{\mathrm{K} 3}$ (which is listed even though $g=3$ ) is assigned weights $(1,1,1,1)$, the weights of a typical example in $\mathbb{P}^{3}$, rather than the weights of $\mathbb{P}^{4}$ above.

In [Brown 06], a computer search with MAGMA found other degenerations of K3 surfaces in codimensions 1 and 2. We reproduce some results of that search in Table 2 as an illustration, but see [Brown 06] for more and for the combination of degeneration and unprojection calculations behind them.

\subsection{Elliptic Fibrations and Shimada's Classification}

By Theorem 2.7, the K3 database contains all K3 surfaces (at least those of genus less than or equal to 2). From now on, we consider the converse: which candidates in the database actually arise as K3 surfaces. We understand two different positive answers.

Definition 2.10. Let $\xi \in \mathcal{D}_{\mathrm{K} 3}$ be a numerical K3 candidate from the $\mathrm{K} 3$ database and $\left(a_{0}, \ldots, a_{N}\right)$ its weights. We say that

(a) $\xi$ represents a K3 Hilbert series if there is a polarized K3 surface $X, A$ with $P_{X}(t)=P_{\xi}(t)$.

(b) $\xi$ represents a K3 surface if there is a polarized K3 surface $X, A$ with $P_{X}(t)=P_{\xi}(t)$ whose graded ring $R(X, A)$ has a generating set $x_{0}, \ldots, x_{N} \in R(X, A)$ that are homogeneous of degrees $\operatorname{deg} x_{i}=a_{i}$.

We consider the stronger statement (b) in Section 3 below. The natural approach to (a) is to apply the Torelli theorem for K3 surfaces; we do not do that here, although 


\begin{tabular}{|c|c|c|c|c|c|c|c|c|c|c|c|c|}
\hline & -1 & 0 & 1 & 2 & 3 & 4 & 5 & 6 & 7 & 8 & 9 & total \\
\hline 1 & 54 & 32 & 6 & 2 & 1 & 0 & 0 & 0 & 0 & 0 & 0 & 95 \\
\hline 2 & 45 & 29 & 6 & 2 & 1 & 1 & 0 & 0 & 0 & 0 & 0 & 84 \\
\hline 3 & 26 & 29 & 8 & 3 & 2 & 1 & 1 & 0 & 0 & 0 & 0 & 70 \\
\hline 4 & 60 & 54 & 15 & 6 & 3 & 2 & 1 & 1 & 0 & 0 & 0 & 142 \\
\hline 5 & 58 & 63 & 21 & 8 & 6 & 3 & 2 & 1 & 1 & 0 & 0 & 163 \\
\hline 6 & 80 & 98 & 35 & 15 & 8 & 6 & 3 & 2 & 1 & 1 & 0 & 249 \\
\hline 7 & 81 & 116 & 49 & 21 & 15 & 8 & 6 & 3 & 2 & 1 & 1 & 303 \\
\hline 8 & 128 & 182 & 79 & 35 & & & & & & & & \\
\hline 9 & 107 & 208 & 109 & 49 & & & & & & & & \\
\hline 10 & 192 & 312 & 171 & 79 & & & & & & & & \\
\hline 11 & 167 & 369 & 236 & 109 & & & & & & & & \\
\hline 12 & 238 & 497 & 353 & 171 & & & & & & & & \\
\hline 13 & 245 & 603 & 488 & 236 & & & & & & & & \\
\hline 14 & 346 & 759 & 720 & 353 & & & & & & & & \\
\hline 15 & 316 & 728 & 982 & 488 & & & & & & & & \\
\hline 16 & 402 & 744 & 1419 & 720 & & & & & & & & \\
\hline 17 & 337 & 581 & 1930 & 982 & & & & & & & & \\
\hline 18 & 350 & 457 & 0 & 1419 & & & & & & & & \\
\hline 19 & 266 & 267 & 0 & 1930 & & & & & & & & \\
\hline 20 & 258 & 171 & 0 & 0 & & & & & & & & \\
\hline 21 & 161 & 85 & 0 & 0 & & & & & & & & \\
\hline 22 & 139 & 55 & 0 & 0 & & & & & & & & \\
\hline 23 & 93 & 24 & 0 & 0 & & & & & & & & \\
\hline 24 & 57 & 13 & 0 & 0 & & & & & & & & \\
\hline 25 & 35 & 3 & 0 & 0 & & & & & & & & \\
\hline 26 & 22 & 0 & 0 & 0 & & & & & & & & \\
\hline 27 & 12 & 0 & 0 & 0 & & & & & & & & \\
\hline 28 & 5 & 0 & 0 & 0 & & & & & & & & \\
\hline 29 & 1 & 0 & 0 & 0 & & & & & & & & \\
\hline total & 4281 & 6479 & 6627 & 6628 & & & & & & & & \\
\hline
\end{tabular}

TABLE 1. Number of $\xi \in \mathcal{D}_{\mathrm{K} 3}$ by codimension (down) and genus (across).

\begin{tabular}{rll}
\hline general member & $\rightsquigarrow$ & degeneration \\
\hline$X_{4} \subset \mathbb{P}(1,1,1,1)$ & $\rightsquigarrow$ & $Y_{2,4} \subset \mathbb{P}(1,1,1,1,2)$ \\
$X_{6} \subset \mathbb{P}(1,1,2,2)$ & $\rightsquigarrow$ & $Y_{3,6} \subset \mathbb{P}(1,1,2,2,3)$ \\
$X_{8} \subset \mathbb{P}(1,2,2,3)$ & $\rightsquigarrow$ & $Y_{4,8} \subset \mathbb{P}(1,2,2,3,4)$ \\
$X_{10} \subset \mathbb{P}(1,2,3,4)$ & $\rightsquigarrow$ & $Y_{5,10} \subset \mathbb{P}(1,2,3,4,5)$ \\
$X_{12} \subset \mathbb{P}(1,2,4,5)$ & $\rightsquigarrow$ & $Y_{6,12} \subset \mathbb{P}(1,2,4,5,6)$ \\
$X_{12} \subset \mathbb{P}(2,3,3,4)$ & $\rightsquigarrow$ & $Y_{6,12} \subset \mathbb{P}(2,3,3,4,6)$ \\
$X_{14} \subset \mathbb{P}(2,3,4,5)$ & $\rightsquigarrow$ & $Y_{7,14} \subset \mathbb{P}(2,3,4,5,7)$ \\
$X_{18} \subset \mathbb{P}(3,4,5,6)$ & $\rightsquigarrow$ & $Y_{9,18} \subset \mathbb{P}(3,4,5,6,9)$ \\
\hline$X_{6} \subset \mathbb{P}(1,1,1,3)$ & $\rightsquigarrow$ & $Y_{2,6} \subset \mathbb{P}(1,1,1,2,3)$ \\
$X_{12} \subset \mathbb{P}(1,2,3,6)$ & $\rightsquigarrow$ & $Y_{4,12} \subset \mathbb{P}(1,2,3,4,6)$ \\
$X_{18} \subset \mathbb{P}(1,3,5,9)$ & $\rightsquigarrow$ & $Y_{6,18} \subset \mathbb{P}(1,3,5,6,9)$ \\
$X_{18} \subset \mathbb{P}(2,3,4,9)$ & $\rightsquigarrow$ & $Y_{6,18} \subset \mathbb{P}(2,3,4,6,9)$ \\
$X_{24} \subset \mathbb{P}(3,4,5,12)$ & $\rightsquigarrow$ & $Y_{8,24} \subset \mathbb{P}(3,4,5,8,12)$ \\
$X_{30} \subset \mathbb{P}(4,5,6,15)$ & $\rightsquigarrow$ & $Y_{10,30} \subset \mathbb{P}(4,5,6,10,15)$ \\
\hline
\end{tabular}

TABLE 2. Some codimension 2 degenerations among the famous 95 .

it is a straightforward computer calculation to confirm that all candidates in codimension up to 6 do at least represent a K3 Hilbert series. Instead, we compare our database with a classification of elliptic K3 surfaces due to Shimada [Shimada 00].
An elliptic K3 surface is a fibration $f: Y \rightarrow \mathbb{P}^{1}$, where $Y$ is a nonsingular K3 surface and the general fiber is a curve of genus 1. (We do not assume that $f$ has a section.) Shimada [Shimada 00] classifies the collections of singular fibers that do appear on elliptic K3 surfaces 
into 3937 different collections (many of which can appear in fibrations having different numbers of sections).

2.3.1 A Nonexistence Result. There are candidates that cannot easily represent a K3 Hilbert series. A polarization $A$ is said to be simple if it intersects the exceptional locus of each singularity transversely at a single point. The candidate in the theorem below is number 76 (of genus 0) in $\mathcal{D}_{\mathrm{K} 3}$.

Theorem 2.11. Let $\xi=(g, \mathcal{B}) \in \mathcal{D}_{\mathrm{K} 3}$ be the candidate with $g=0$ and $\mathcal{B}=\left\{\frac{1}{2}(1,1), 2 \times \frac{1}{10}(1,9)\right\}$. Then there does not exist a polarized $K 3$ surface $X, A$ with $P_{X}(t)=$ $P_{\xi}(t)$ for which the polarization is simple.

Proof: Suppose $X, A$ is a polarized K3 surface with $P_{X}(t)=P_{\xi}(t)$. We have $H^{0}(X, A)=1$ since $g=0$, so we may regard $A \subset X$ as an effective divisor with $A^{2}=-2$. Let $\varphi: Y \rightarrow X$ be the minimal resolution of singularities; $Y$ is a nonsingular K3 surface. We estimate the rank of the Picard group $\operatorname{Pic}(Y)$.

In the group $\operatorname{Pic}(Y)$, the exceptional curves of $\varphi$ are independent of one another and of the components of A. The exceptional curves will generate a subgroup of rank 19 if the singularities of $X$ are exactly those of the basket. The list of possible degenerations of a basket in display (2-4) shows that that if the singularities of $X$ are not those of the basket, then the exceptional curves will generate a subgroup of rank at least 20 . Since the Picard rank of a K3 surface is at most 20, we conclude that the singularities of $X$ are those of its basket and that $A$ is an irreducible rational curve. Otherwise, its components would also contribute independently to a rank exceeding 20 .

So, since the polarization is simple, the configuration of 20 nonsingular rational curves, each with self intersection -2 , pictured in Figure 1, lies on $Y$. The divisor

$$
2 B+4 A+3 C_{1}+2 C_{2}+C_{3}+3 D_{1}+2 D_{2}+D_{3}
$$

is an elliptic fiber $\widetilde{\mathrm{E}}_{7}$ on $Y$. This fiber generates an elliptic fibration on $Y$ with at least two sections (being the two exceptional curves $C_{4}, D_{4}$ adjacent to the $\widetilde{\mathrm{E}}_{7}$ configuration). The remaining exceptional curves must be contained in other elliptic fibers. Thus the only possibilities for the singular fibers of this fibration are $\widetilde{\mathrm{E}}_{7}+\widetilde{\mathrm{A}}_{5}+\widetilde{\mathrm{A}}_{5}$, $\widetilde{\mathrm{E}}_{7}+\widetilde{\mathrm{A}}_{5}+\widetilde{\mathrm{A}}_{5}+\widetilde{\mathrm{A}}_{1}$, and $\widetilde{\mathrm{E}}_{7}+\widetilde{\mathrm{A}}_{11}$. Such combinations of elliptic fibers do occur according to Shimada's classification [Shimada 00], but they occur only with no sections at all or with exactly one section. So $Y$ cannot exist as a K3 surface, and so neither does $X$.

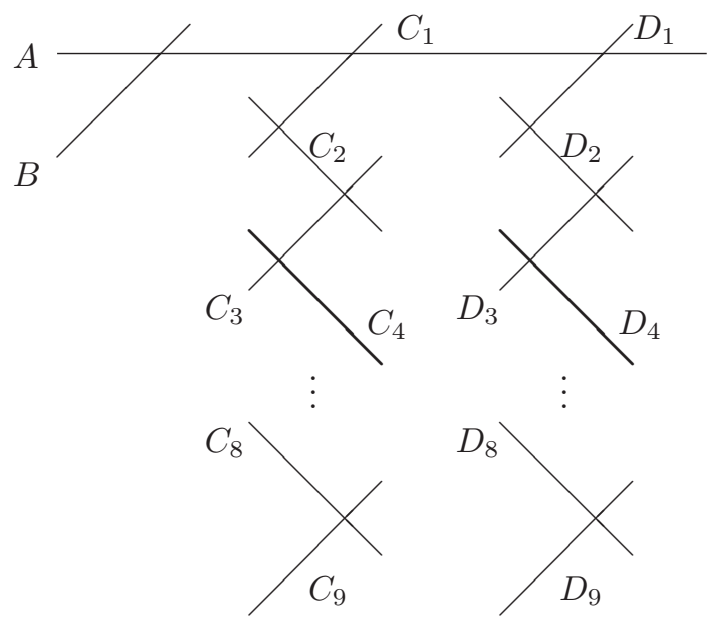

FIGURE 1. A configuration of curves on $Y$.

2.3.2 Realizing a Hilbert Series. In a closely related example, let $f: Y \rightarrow \mathbb{P}^{1}$ be the elliptic K3 surface number 3305 in Shimada's classification. It has two singular fibers, of types $\widetilde{\mathrm{E}}_{7}$ and $\widetilde{\mathrm{A}}_{11}$ respectively, and was one of the cases considered in the proof of Theorem 2.11. Furthermore, the Mordell-Weil group of $f$ contains exactly one element that is the unique section of $f$. Therefore, the K3 surface $Y$ contains the configuration of -2-curves pictured in Figure 2.

We define a $\mathbb{Q}$-divisor $B$ on $Y$ supported on this configuration of curves:

$$
\begin{aligned}
B= & \frac{1}{16}\left(E_{1}+2 E_{2}+\cdots+15 E_{15}\right)+E_{16} \\
& +\frac{1}{4}\left(3 E_{17}+2 E_{18}+E_{19}\right)+\frac{1}{2} E_{20} .
\end{aligned}
$$

It is easy to check that $B$ is $\mathbb{Q}$-ample (modulo some - 2curves in its support on which it is trivial) and that some multiple of $B$ gives a morphism $\varphi$ of $Y$ to some projective

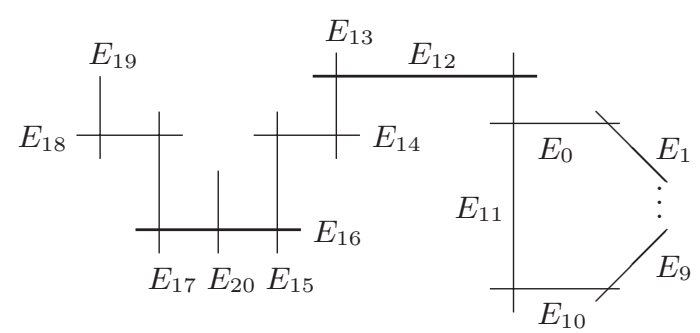

FIGURE 2. Shimada's elliptic fibration number 3305. Section of the fibration is $E_{12}$. Fiber $\widetilde{\mathrm{A}}_{11}$ is $E_{0}+E_{1}+\cdots+E_{11}$. Fiber $\widetilde{\mathrm{E}}_{7}$ is $\left(E_{13}+E_{19}\right)+2\left(E_{14}+E_{18}+E_{20}\right)+3\left(E_{15}+E_{17}\right)+$ $4 E_{16}$. 
space that is birational to its image and contracts all of the curves $E_{i}$ of the configuration except $E_{0}$ and $E_{16}$. Let $X=\varphi(Y)$ and define $A$ to be the (integral) divisor $\varphi_{*}(B)$ on $X$. Again, it is easy to check that $A^{2}=3 / 16$ and that the basket $\mathcal{B}$ and genus $g$ of $X, A$ are

$$
\mathcal{B}=\left\{\frac{1}{2}(1,1), \frac{1}{4}(1,3), \frac{1}{16}(1,15)\right\} \quad \text { and } \quad g=0 .
$$

Indeed, $\mathcal{D}_{\mathrm{K} 3}$ contains such a numerical $\mathrm{K} 3$ candidate $\xi=(g, \mathcal{B})$. In the $\mathrm{K} 3$ database it is number 35 (of genus 0 ), where it is described provocatively as

$$
X \subset \mathbb{P}^{12}(1,4,6,7,8,9,10,11,12,13,14,15,16) .
$$

We conclude that $\xi$ represents a K3 Hilbert series. But bear in mind what we are not claiming: while this description is meaningful, one cannot conclude that there really is a quasismooth K3 surface in this wps that realizes $\xi$.

\section{NUMERICAL UNPROJECTION AND WEIGHTS}

Theorem 2.11 shows that there are candidates in the database that might not even represent a K3 Hilbert series, let alone a K3 surface. Even so, here we attempt to compute plausible weights for every $\xi \in \mathcal{D}_{\mathrm{K} 3}$ as a first step toward the stronger statement (b).

The results of Reid, Iano-Fletcher [Iano-Fletcher 00], and Altinok [Altinok 98] imply that every $\xi \in \mathcal{D}_{\mathrm{K} 3}$ with codimension at most 3 represents a K3 surface. Altmok also uses unprojection methods to show that the majority of $\xi$ with codimension 4 represent a K3 surface, which Frantzen [Frantzen 04] extends to confirm the same for some in codimension 5. We use unprojection methods to make predictions of weights in higher codimension - in the precise sense of Computation 3.5 below - although we cannot carry out the calculations to confirm that every $\xi \in \mathcal{D}_{\mathrm{K} 3}$ represents a K3 surface.

\subsection{Type I and Type $\mathrm{II}_{n}$ unprojections}

3.1.1 Kustin-Miller Unprojections. Type-I unprojection, also known as Kustin-Miller unprojection, is a general operation that constructs bigger Gorenstein rings from smaller ones. We use it to mean the map $X \rightarrow Y$ in the following theorem.

Theorem 3.1. (Papadakis-Reid.) [Papadakis and Reid 04] Let $X, A$ be a polarized K3 surface and $X \subset$ $\mathbb{P}\left(a_{0}, \ldots, a_{N}\right)$ its embedding by $A$. Suppose that $X$ contains the coordinate line $C=\mathbb{P}\left(a_{i}, a_{j}\right)$ and that $X$ is quasismooth along $C$ in this embedding. Then (a) There is a K3 surface $Y \subset \mathbb{P}\left(a_{0}, \ldots, a_{N}, a_{i}+a_{j}\right)$ containing the coordinate point $P_{N+1}=(0, \ldots, 0,1)$.

(b) The Gorenstein projection of $Y$ from $P_{N+1}$ is a birational map $Y \rightarrow X$ with exceptional sets $C \subset X$ and $P_{N+1} \in Y$. The birational inverse $X \rightarrow Y$ is the contraction of the -2 -curve $C \subset X$.

(c) If $a_{i}+a_{j}+a_{k}>a_{\ell}$ for every $k, \ell \in\{0, \ldots, N\} \backslash\{i, j\}$, then the unprojection equations of $Y$ embedded in $\mathbb{P}\left(a_{0}, \ldots, a_{N}, a_{i}+a_{j}\right)$ have no linear terms.

(d) $Y$ is polarized by $B=A+\frac{1}{a_{i}+a_{j}} C$, and its Hilbert series is

$$
P_{Y, B}(t)=P_{X, A}(t)+\frac{t^{a_{i}+a_{j}}}{\left(1-t^{a_{i}+a_{j}}\right)\left(1-t^{a_{i}}\right)\left(1-t^{a_{j}}\right)} .
$$

In particular, the genus of $Y, B$ equals the genus of $X, A$.

(e) Let $\mathcal{B}_{Y}$ be the basket of $Y$ and $\mathcal{B}_{X}$ that of $X$. Then

$$
\begin{aligned}
\mathcal{B}_{X} \cup & \left\{\frac{1}{a_{i}+a_{j}}\left(a_{i}, a_{j}\right)\right\} \\
& =\mathcal{B}_{Y} \cup\left\{\frac{1}{a_{i}}\left(a_{j},-a_{j}\right), \frac{1}{a_{j}}\left(a_{i},-a_{i}\right),\right\},
\end{aligned}
$$

where any singularity type $\frac{1}{r}(a,-a)$ of index $r=1$ can be omitted.

The proof of most of this theorem is given (or implicit) across a number of sources including [Papadakis and Reid 04, Altınok 98, Altınok et al. 02, Frantzen 04], so we sketch a proof here for convenience using only the main theorem of [Papadakis and Reid 04].

Proof: The setup $C \subset X$ is of a Type-I unprojection: the ideals of $X \subset \mathbb{P}^{N}$ and $C \subset \mathbb{P}^{N}$ are Gorenstein. It follows by [Papadakis and Reid 04, Theorem 1] that there is a rational form $s \in \mathbb{C}(\bar{X})$ on the affine cone $\bar{X}$ of $X$, unique up to scalar multiple and of weight $a_{i}+a_{j}$, that has a simple pole along $C$. The extension of algebras $R(X, A) \subset R(X, A)[s]$ inside $\mathbb{C}(\bar{X})$ defines a birational map of varieties $X \rightarrow Y$ contracting exactly $C$. Since $X$ is quasismooth, this map is a morphism, and so it is the contraction of a -2-curve on $X$, and $X$ is a partial resolution of $Y$. In particular, the resulting $Y$ is again a K3 surface.

If $x_{0}, \ldots, x_{N}$ are given generators of $R(X, A)$ of degrees $\operatorname{deg} x_{i}=a_{i}$, we extend this list by $x_{N+1}=s$ to give an embedding of $Y$ as in (a). Eliminating $s$ from 
the coordinate ring of $Y$ in this embedding recovers $X$, which is (b).

According to the recipe of [Papadakis and Reid 04, Theorem 1], the unprojection equations are of the form

$$
x_{N+1} x_{k}=g_{k} \text { for } k \neq i, j \text { and some } g_{k} \in R(X, A) \text {. }
$$

These equations have degree $a_{i}+a_{j}+a_{k}$ for $k$ in the set $\{0, \ldots, N\} \backslash\{i, j\}$. The condition given in (c) is that this degree is higher than that of any of the variables, and so these variables cannot appear linearly.

The divisor of $s$ on $X$ contains $C$ with coefficient -1 and is linearly equivalent to $\left(a_{i}+a_{j}\right) A$. So expressed as a divisor on $X$ before contracting $C$, the hyperplane section of $Y$ is $B$ as stated in (d). The formula computes the number of monomials added to $R(X, A)$ in each degree by the inclusion of $s$. It counts multiples of $s$ by the variables $x_{i}, x_{j}$ and by $s$ itself. All other monomials $s x_{k}$ can be eliminated by the unprojection equations.

Finally, (e) follows from the formula in (d) together with Theorem 2.1. Alternatively, one sees from the unprojection equations that $P_{N+1} \in Y$ is of type $\frac{1}{a_{i}+a_{j}}\left(a_{i}, a_{j}\right)$, which forces the quotient singularities of $X$ along $C$ to be the stated pair.

In principle, this theorem provides an inductive framework for generating K3 surfaces in high codimension to realize items in $\mathcal{D}_{\mathrm{K} 3}$. Indeed, this is how Altınok [Altınok 98] and Frantzen [Frantzen 04] construct K3 surfaces in codimensions 4 and 5 . However, verifying that such $X \supset C$ exist and are quasismooth seems difficult in general. Instead, we use the theorem to generate plausible weights for any $\xi \in \mathcal{D}_{\mathrm{K} 3}$ as follows.

Definition 3.2. For $\eta=(g, \mathcal{B}) \in \mathcal{D}_{\mathrm{K} 3}$ and $p=\frac{1}{r}(a,-a) \in$ $\mathcal{B}$, the numerical projection of $p$ in $\eta$ is $\xi=\left(g, \mathcal{B}^{\prime}\right)$, where

$$
\mathcal{B}^{\prime} \cup\left\{\frac{1}{r}(a,-a)\right\}=\mathcal{B} \cup\left\{\frac{1}{a}(r,-r), \frac{1}{r-a}(r,-r)\right\},
$$

and we omit any singularity of type $\frac{1}{s}(c,-c)$ of index $s=1$.

Algorithm 3.3. (Type-I forcing.) For fixed genus $-1 \leq$ $g \leq 2$, let $\mathcal{D}_{\mathrm{K} 3}(g)$ be the subset of $\mathcal{D}_{\mathrm{K} 3}$ of numerical $\mathrm{K} 3$ candidates with genus $g$ ordered in increasing Hilbert series order.

For $\eta=(g, \mathcal{B}) \in \mathcal{D}_{\mathrm{K} 3}(g)$ do

(1) If $P_{\eta}$ is that of a known K3 surface of codimension less than or equal to 2 , then assign it these known weights; continue with the next $\eta$.
(2) For each $p=\frac{1}{r}(a,-a) \in \mathcal{B}$ :

(a) Compute the numerical projection $\xi$ of $p$ in $\eta$.

(b) If $(g, \xi) \in \mathcal{D}_{\mathrm{K} 3}(g)$ and the pair $a, r-a$ occurs among the weights $W$ of $\xi$, then let the weights of $\xi$ be $W \cup\{r\}$; continue with the next $\eta$.

(3) Apply the naive algorithm of Section 2.2 to generate weights for $\eta$; continue with the next $\eta$.

In (1), we take the $95+84 \mathrm{~K} 3$ surfaces in codimension at most 2 as known. Of course, in $(2)(\mathrm{b})$ the pair $(g, \xi)$ will be in $\mathcal{D}_{\mathrm{K} 3}$ if and only if $A_{\xi}^{2}>0$, and furthermore, the weights of $\xi$ will be known inductively because $\xi$ appears in $\mathcal{D}_{\mathrm{K} 3}(g)$ ahead of $\eta$ by the Hilbert series order together with Theorem 3.1(d).

Part (3) of the algorithm is not very satisfactory. One can improve on the naive algorithm by adding other weights to realize the basket better, for instance. But we don't discuss that here, since in practice, the unprojection step (2) is enough once higher unprojections are included.

3.1.2 Higher Unprojections. Type-I unprojections are only one kind of unprojection calculation associated with Gorenstein rings. At the time of writing, it is the only one for which the theory is complete, although $\mathrm{Pa}-$ padakis [Papadakis 06] has recently proved some results for Type II. However, it is still possible to calculate with other kinds. Experience from examples suggests the following numerical characterization of another type of unprojection.

Definition 3.4. Suppose $\xi \in \mathcal{D}_{\mathrm{K} 3}$ is a numerical projection of $\eta$, that is, $\xi$ and $\eta$ are related as in Definition 3.2. Let $W_{\xi}$ be the weights of $\xi$ and let $n$ be a positive integer. Then the projection is a numerical Type-IIn projection if $(r-a) \in W_{\xi}$ and $n+1$ is the smallest positive integer $k$ for which $k a \in W_{\xi} \backslash\{r-a\}$ (or the analogous statement with $a$ and $r-a$ switching roles).

In this situation and notation, we define the expected weights of $\eta$ to be

$$
W_{\eta}=W_{\xi} \cup\{r, r+a, r+2 a, \ldots, r+n a\} .
$$

The idea is that the new weight $r$ corresponds to an unprojection variable $s$ as for Type I, but that additional variables are needed to make the unprojection projectively normal. 
For example, projecting from

$$
\frac{1}{2}(1,1) \in Y \subset \mathbb{P}^{5}(1,2,2,3,5,7)
$$

is a numerical Type- $\mathrm{II}_{1}$ projection with image

$$
\mathbb{P}^{1} \subset X_{15} \subset \mathbb{P}^{3}(1,2,5,7) .
$$

A single variable of weight 2 has been eliminated, and with it the variable of weight 3 that polarized the singularity is also eliminated. One could eliminate the weight3 variable alone to see the nonnormal unprojection. See Section 4 for this projection in the database.

We can force Type-II unprojections just as for Type I in Algorithm 3.3. In constructing $\mathcal{D}_{\mathrm{K} 3}$, exactly this is done using Types $\mathrm{II}_{1}$ and $\mathrm{II}_{2}$ once the possibility of a Type-I unprojection has been exhausted. Once constructed, the whole K3 database is subjected to the following consistency check.

Computation 3.5. (Numerical Type-I and -II Consistency.) The weights of numerical K3 candidates in $\mathcal{D}_{\mathrm{K} 3}$ are consistent with all projections of numerical Type I and Type $\mathrm{II}_{n}$ for any $n>0$ from any candidate of codimension 3 or more.

The proof is a computer calculation: For each $\xi \in \mathcal{D}_{\mathrm{K} 3}$ that does not correspond to a known K3 surface in codimension 1 or 2, the weights of $\xi$ are computed according to every projection of numerical Types I and II, and the results are required to be the same. Candidates in codimension 1 or 2 are ignored, since they are already known to be correct (and projection can be more complicated in such small graded rings).

This computation is important. Together with the few hundred initial cases, it is the main supporting evidence that the families described by the database do represent K3 surfaces in the sense of Definition 2.10(b).

Meaning 3.6. The weights associated with numerical K3 candidates in $\mathcal{D}_{\mathrm{K} 3}$ are consistent with the existence of Type-I and -II unprojections between K3 surfaces realizing them.

One could use the same unprojection calculus to predict weights for any $(g, \mathcal{B})$ with $g \geq 3$ that is not listed in $\mathcal{D}_{\mathrm{K} 3}$. The results would be the same as those for $(2, \mathcal{B})$, but with the inclusion of the weight 1 an additional $g-2$ times. Such continuation of $\mathcal{D}_{\mathrm{K} 3}$ would be visible in Table 1 as the $g=2$ column copied in each column to the right, but in higher codimension at each higher genus (with the two candidates in minimal codimension put in codimensions $g-1$ and $g-2$, as at the head of the $g=3$ column).

\subsection{K3 Surfaces Admitting No Gorenstein Projection}

Since the existence of projections is the basis for the computation of higher-codimension weights in $\mathcal{D}_{\mathrm{K} 3}$, the following result limiting those numerical K3 candidates having no projections, or only projections not of numerical Type I or II, is important.

Computation 3.7. Let $\xi=(g, \mathcal{B}) \in \mathcal{D}_{\mathrm{K} 3}$ be a numerical K3 candidate.

If $g \leq 2$ and $\xi$ does not have any numerical projection to another polarized K3 surface, then $\xi$ is one of the following:

\begin{aligned}$g & \xi \\$\hline-1 & codim $\xi \leq 4$ and $\xi$ is one of 36 cases of Table 3 \\ 0 & codim $\xi=1$ and $\xi$ is one of 6 cases of Table 4 \\ 1 & numerical data of $X_{12} \subset \mathbb{P}(1,1,4,6) \\ 2 &$ numerical data of $X_{6} \subset \mathbb{P}(1,1,1,3)\end{aligned}$

If $\xi$ has at least one numerical projection but does not have a numerical projection of Type I or II, then $g=-1$ and $\xi$ is of the form

$$
X \subset \mathbb{P}^{7+3 k}\left(2^{4+k}, 3^{4+2 k}\right) \quad \text { for } k=0, \ldots, 6
$$

(where $2^{n}$ indicates $n$ occurrences of weight 2 ) with $\mathcal{B}=$ $\left\{(10+k) \times \frac{1}{2}(1,1)\right\}$ and $A^{2}=(k+2) / 2$, or $\xi$ is one of the following three cases:

$$
\begin{gathered}
X \subset \mathbb{P}^{8}(8,8,9,10,11,12,13,14,15), \\
A^{2}=\frac{1}{2}+\frac{3}{4}+\frac{3 \cdot 5}{8}+\frac{8}{9}=\frac{1}{72} ; \\
X \subset \mathbb{P}^{7}(7,7,8,9,10,11,12,13) \\
\quad A^{2}=\frac{2 \cdot 5}{7}+\frac{3 \cdot 4}{7}+\frac{7}{8}=\frac{1}{56} ; \\
X \subset \mathbb{P}^{5}(4,5,5,6,7,8), \\
\quad A^{2}=\frac{1}{2}+\frac{3}{4}+2 \times \frac{4}{5}+\frac{2 \cdot 3}{5}=\frac{1}{20} ;
\end{gathered}
$$

where $\frac{1}{r}(a,-a) \in \mathcal{B}$ is represented by its contribution $b(r-b) / r$ to $A^{2}$ in $(2-3)$.

The first half of this computation already appeared in [Brown 03]. The second half can be computed from the K3 database using code similar to that of section 4 .

\section{USING THE K3 DATABASE}

There are three ways to access the K3 database. The main one is to use Magma, as described below. Second, the website [Brown et al. 04] has a bureaucratic 


\begin{tabular}{|c|c|c|c|c|c|}
\hline & & K3 surface & $\rho_{X}$ & Basket $\mathcal{B}$ & Degree $A^{2}$ \\
\hline$X_{50}$ & $\subset$ & $\mathbb{P}(7,8,10,25)$ & 19 & $\frac{1}{2}+\frac{2 \cdot 3}{5}+\frac{2 \cdot 5}{7}+\frac{7}{8}$ & $1 / 280$ \\
\hline$X_{36}$ & $\subset$ & $\mathbb{P}(7,8,9,12)$ & 19 & $\frac{2}{3}+\frac{3}{4}+\frac{3 \cdot 4}{7}+\frac{7}{8}$ & $1 / 168$ \\
\hline$X_{40}$ & $\subset$ & $\mathbb{P}(5,7,8,20)$ & 18 & $\frac{3}{4}+2 \times \frac{2 \cdot 3}{5}+\frac{6}{7}$ & $1 / 140$ \\
\hline$X_{66}$ & $\subset$ & $\mathbb{P}(5,6,22,33)$ & 18 & $\frac{1}{2}+\frac{2}{3}+\frac{2 \cdot 3}{5}+\frac{2 \cdot 9}{11}$ & $1 / 330$ \\
\hline$X_{38}$ & $\subset$ & $\mathbb{P}(5,6,8,19)$ & 18 & $-\frac{4}{5}+\frac{5}{6}+\frac{3 \cdot 5}{8}$ & $1 / 120$ \\
\hline$X_{27}$ & $\subset$ & $\mathbb{P}(5,6,7,9)$ & 18 & $\frac{2}{3}+\frac{4}{5}+\frac{5}{6}+\frac{3 \cdot 4}{7}$ & $1 / 70$ \\
\hline$X_{34}$ & $\subset$ & $\mathbb{P}(4,6,7,17)$ & 17 & $2 \times \frac{1}{2}+\frac{3}{4}+\frac{5}{6}+\frac{3 \cdot 4}{7}$ & $1 / 84$ \\
\hline$X_{54}$ & $\subset$ & $\mathbb{P}(4,5,18,27)$ & 17 & $\frac{1}{2}+\frac{3}{4}+\frac{2 \cdot 3}{5}+\frac{2 \cdot 7}{9}$ & $1 / 180$ \\
\hline$X_{32}$ & $\subset$ & $\mathbb{P}(4,5,7,16)$ & 17 & $2 \times \frac{3}{4}+\frac{4}{5}+\frac{3 \cdot 4}{7}$ & $1 / 70$ \\
\hline$X_{30}$ & $\subset$ & $\mathbb{P}(4,5,6,15)$ & 16 & $2 \times \frac{1}{2}+\frac{2}{3}+\frac{3}{4}+2 \times \frac{4}{5}$ & $1 / 60$ \\
\hline$X_{24}$ & $\subset$ & $\mathbb{P}(3,6,7,8)$ & 16 & $\frac{1}{2}+4 \times \frac{2}{3}+\frac{6}{7}$ & $1 / 42$ \\
\hline$X_{48}$ & $\subset$ & $\mathbb{P}(3,5,16,24)$ & 16 & $2 \times \frac{2}{3}+\frac{4}{5}+\frac{3 \cdot 5}{8}$ & $1 / 120$ \\
\hline$X_{21}$ & $\subset$ & $\mathbb{P}(3,5,6,7)$ & 16 & $3 \times \frac{2}{3}+\frac{2 \cdot 3}{5} 23+\frac{5}{6}$ & $1 / 30$ \\
\hline$X_{42}$ & $\subset$ & $\mathbb{P}(3,4,14,21)$ & 15 & $\frac{1}{2}+2 \times \frac{2}{3}+\frac{3}{4}+\frac{2 \cdot 5}{7}$ & $1 / 84$ \\
\hline$X_{24}$ & $\subset$ & $\mathbb{P}(3,4,5,12)$ & 15 & $2 \times \frac{2}{3}+2 \times \frac{3}{4}+\frac{2 \cdot 3}{5}$ & $1 / 30$ \\
\hline$X_{18}$ & $\subset$ & $\mathbb{P}(3,4,5,6)$ & 15 & $\frac{1}{2}+3 \times \frac{2}{3}+\frac{3}{4}+\frac{4}{5}$ & $1 / 20$ \\
\hline$X_{15}$ & $\subset$ & $\mathbb{P}(3,3,4,5)$ & 14 & $5 \times \frac{2}{3}+\frac{3}{4}$ & $1 / 12$ \\
\hline$X_{30}$ & $\subset$ & $\mathbb{P}(2,6,7,15)$ & 14 & $5 \times \frac{1}{2}+\frac{2}{3}+\frac{6}{7}$ & $1 / 42$ \\
\hline$X_{42}$ & $\subset$ & $\mathbb{P}(2,5,14,21)$ & 14 & $3 \times \frac{1}{2}+\frac{4}{5}+\frac{3 \cdot 4}{7}$ & $1 / 70$ \\
\hline$X_{26}$ & $\subset$ & $\mathbb{P}(2,5,6,13)$ & 14 & $4 \times \frac{1}{2}+\frac{2 \cdot 3}{5}+\frac{5}{6}$ & $1 / 30$ \\
\hline$X_{22}$ & $\subset$ & $\mathbb{P}(2,4,5,11)$ & 13 & $5 \times \frac{1}{2}+\frac{3}{4}+\frac{4}{5}$ & $1 / 20$ \\
\hline$X_{30}$ & $\subset$ & $\mathbb{P}(2,3,10,15)$ & 12 & $3 \times \frac{1}{2}+2 \times \frac{2}{3}+\frac{2 \cdot 3}{5}$ & $1 / 30$ \\
\hline$X_{18}$ & $\subset$ & $\mathbb{P}(2,3,4,9)$ & 12 & $4 \times \frac{1}{2}+2 \times \frac{2}{3}+\frac{3}{4}$ & $1 / 12$ \\
\hline$X_{12}$ & $\subset$ & $\mathbb{P}(2,3,3,4)$ & 12 & $3 \times \frac{1}{2}+4 \times \frac{2}{3}$ & $1 / 6$ \\
\hline$X_{14}$ & $\subset$ & $\mathbb{P}(2,2,3,7)$ & 10 & $7 \times \frac{1}{2}+\frac{2}{3}$ & $1 / 6$ \\
\hline$X_{24,30}$ & $\subset$ & $\mathbb{P}(8,9,10,12,15)$ & 19 & $\frac{1}{2}+\frac{2}{3}+\frac{3}{4}+\frac{2 \cdot 3}{5}+\frac{8}{9}$ & $1 / 180$ \\
\hline$X_{18,30}$ & $\subset$ & $\mathbb{P}(6,8,9,10,15)$ & 18 & $2 \times \frac{1}{2}+2 \times \frac{2}{3}+\frac{4}{5}+\frac{7}{8}$ & $1 / 120$ \\
\hline$X_{16,18}$ & $\subset$ & $\mathbb{P}(4,6,7,8,9)$ & 17 & $2 \times \frac{1}{2}+\frac{2}{3}+2 \times \frac{3}{4}+\frac{6}{7}$ & $1 / 42$ \\
\hline$X_{14,16}$ & $\subset$ & $\mathbb{P}(4,5,6,7,8)$ & 17 & $\frac{1}{2}+2 \times \frac{3}{4}+\frac{2 \cdot 3}{5}+\frac{5}{6}$ & $1 / 30$ \\
\hline$X_{12,14}$ & $\subset$ & $\mathbb{P}(4,4,5,6,7)$ & 16 & $2 \times \frac{1}{2}+3 \times \frac{3}{4}+\frac{4}{5}$ & $1 / 20$ \\
\hline$X_{10,12}$ & $\subset$ & $\mathbb{P}(3,4,4,5,6)$ & 15 & $\frac{1}{2}+2 \times \frac{2}{3}+3 \times \frac{3}{4}$ & $1 / 12$ \\
\hline$X_{6,6}$ & $\subset$ & $\mathbb{P}(2,2,2,3,3)$ & 10 & $9 \times \frac{1}{2}$ & $1 / 2$ \\
\hline$X_{16}, \ldots, 20$ & $\subset$ & $\mathbb{P}(5,6,7,8,9,10)$ & 18 & $\frac{1}{2}+\frac{2}{3}+\frac{4}{5}+\frac{2 \cdot 3}{5}+\frac{6}{7}$ & $1 / 42$ \\
\hline$X_{14, \ldots, 18}$ & $\subset$ & $\mathbb{P}(5,5,6,7,8,9)$ & 18 & $\frac{4}{5}+2 \times \frac{2 \cdot 3}{5}+\frac{5}{6}$ & $1 / 30$ \\
\hline$X$ & $\subset$ & $\mathbb{P}(6,6,7,8,9,10,11)$ & 18 & $2 \times \frac{1}{2}+2 \times \frac{2}{3}+\frac{5}{6}+\frac{6}{7}$ & $1 / 42$ \\
\hline$X$ & $\subset$ & $\mathbb{P}(5,6,6,7,8,9,10)$ & 18 & & $1 / 30$ \\
\hline
\end{tabular}

TABLE 3. K3 surfaces with $g=-1$ having no Gorenstein projection.

\begin{tabular}{ccccc} 
& $\mathrm{K} 3$ surface & $\rho_{X}$ & Basket $\mathcal{B}$ & Degree $A^{2}$ \\
\hline$X_{42}$ & $\subset \mathbb{P}(1,6,14,21)$ & 10 & $\frac{1}{2}+\frac{2}{3}+\frac{6}{7}$ & $1 / 42$ \\
$X_{36}$ & $\subset \mathbb{P}(1,5,12,18)$ & 10 & $\frac{2 \cdot 3}{5}+\frac{5}{6}$ & $1 / 30$ \\
$X_{30}$ & $\subset \mathbb{P}(1,4,10,15)$ & 9 & $\frac{1}{2}+\frac{3}{4}+\frac{4}{5}$ & $1 / 20$ \\
$X_{24}$ & $\subset \mathbb{P}(1,3,8,12)$ & 8 & $2 \times \frac{2}{3}+\frac{3}{4}$ & $1 / 12$ \\
$X_{18}$ & $\subset \mathbb{P}(1,2,6,9)$ & 6 & $3 \times \frac{1}{2}+\frac{2}{3}$ & $1 / 6$ \\
$X_{10}$ & $\subset \mathbb{P}(1,2,2,5)$ & 6 & $5 \times \frac{1}{2}$ & $1 / 2$ \\
\hline
\end{tabular}

TABLE 4. K3 surfaces with $g=0$ having no Gorenstein projection.

front end to Magma with a form to fill in that can be submitted to the K3 database. And third, there is an SQL-style version of the database posted on the website [Brown et al. 04] that can be downloaded and installed under an SQL server. Of course, this is static and does not include some data that is computed live by MAGMA.

\subsection{The K3 Database in MAGMA}

The computer algebra system MAgMA [Cannon 05, Bosma et al. 97] (version 2.11 or higher) contains a database of 24,099 representative K3 surfaces. We give an example of a continuous session using this database. Having already started MAGMA (typically by typing magma at a command line), we name the K3 database $D$. 
$>\mathrm{D}:=$ K3Database () ;

$>\mathrm{D}$;

The database of K3 surfaces

Now we pick out a surface $X$ with given weights. It can be analyzed using various function calls like Degree(X). But simply printing it on screen presents all of its useful data.

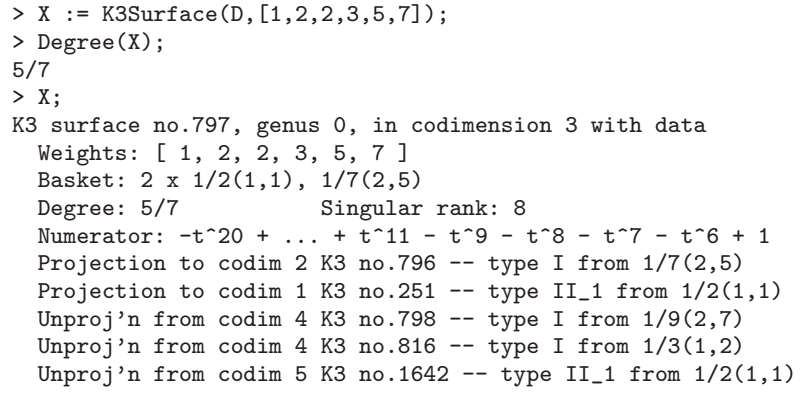

In this case, reading the numerator suggests that there are four equations of weights $6,7,8,9$ respectively. In fact, it is known that Gorenstein rings in codimension 3 have an odd number of relations, so one guesses that there are five equations, the missing one being in degree 10 , masked in the numerator by some syzygy also of degree 10. This now works, and one can write $X$ as the five maximal Pfaffians of a skew $5 \times 5$ matrix; compare with [Altinok et al. 02, Example 3.7] .

The weights of $X$ can be deduced using a Type-I unprojection. Indeed, we see that the $g=0$ surface number 796 has the right numerical properties to be a Type-I projection from the $\frac{1}{7}(2,5)$ point of $X$.

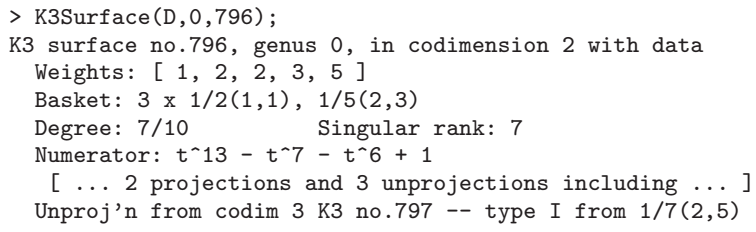

Indeed, this can be realized, and the unprojection can be calculated; compare again [Altinok et al. 02, Example $3.7]$.

The surface $X$ has a second projection. By Computation 3.5, the weights of $X$ can be calculated using this projection instead and should give the same result. Indeed, looking at the image of the numerical projection, we see that its weights differ only by the missing pair 2,3 , which is also the prediction using the numerical Type- $\mathrm{II}_{1}$ projection of Definition 3.4.

$>\operatorname{K3Surface}(\mathrm{D}, 0,251)$;

K3 surface no.251, genus 0 , in codimension 1 with data

Weights: [ $1,2,5,7$ ]

Basket: $1 / 2(1,1), 1 / 7(2,5)$

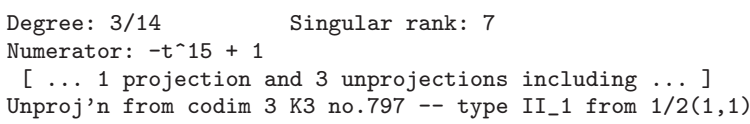

One can make more serious searches, testing predicates on each surface in the database. For example, we make a sequence containing those codimension-5 surfaces with no unprojections; there is only one of them.

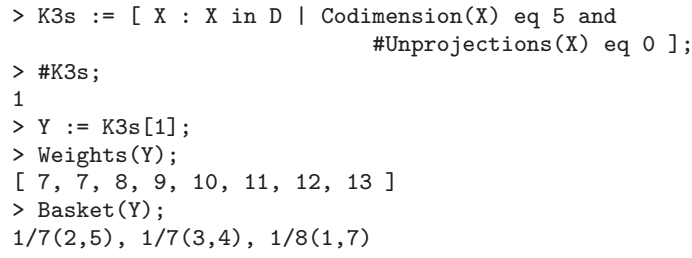

Or we can confirm the lower bound in Computation 1.1: $X_{66} \subset \mathbb{P}(5,6,22,33)$ has degree $1 / 330$.

$>[\operatorname{Weights}(\mathrm{X})$ : X in D | Degree(X) le 1/330 ];

$[[5,6,22,33]]$

To give a typical calculation, we write a function to list all projections of the K3 surface number 35 constructed in Section 2.3.

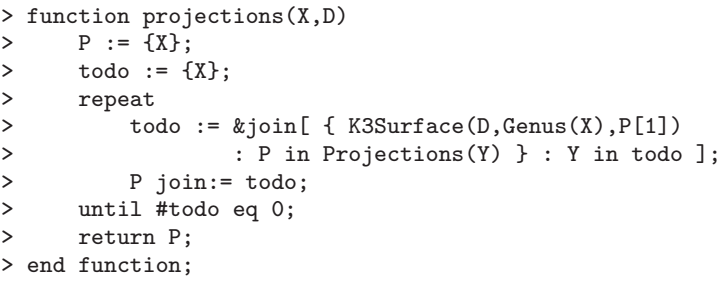

We apply this function to $X$ and look at the codimensions of its projections.

$>\mathrm{X}:=\operatorname{K} 3 \operatorname{Surface}(\mathrm{D}, 0,35)$;

$>\{*$ Codimension $(\mathrm{Y}): \mathrm{Y}$ in projections $(\mathrm{X}, \mathrm{D}) *\}$;

$\left\{* 1^{\wedge} 6,2^{-\cdots} 2,3^{-\sim} 2,4^{\wedge-2}, 5^{\wedge} 2,6^{\wedge} 2,7^{\sim} 2,8^{\cdots} 2,9,10 *\right\}$

In other words, we have the following computation.

Computation 4.1. Let $\xi=(g, \mathcal{B}) \in \mathcal{D}_{\mathrm{K} 3}$ be the numerical $\mathrm{K} 3$ candidate of display $(2-5)$ in Section 2.3. The set of projections of $\xi$ consists of 22 candidates in $\mathcal{D}_{\mathrm{K} 3}$ whose weights are spread across codimensions 1 to 10 as follows:

$$
\begin{array}{c|cccccccccc}
\text { codim } & 1 & 2 & 3 & 4 & 5 & 6 & 7 & 8 & 9 & 10 \\
\hline \text { number } & 6 & 2 & 2 & 2 & 2 & 2 & 2 & 2 & 1 & 1
\end{array} .
$$

Since $\xi$ represents a K3 Hilbert series, so does each of these 22 candidates.

\section{ACKNOWLEDGMENTS}

This paper was written at the University of Warwick under EPSRC grant GR/S03980/01 Algebraic geometry, graded rings and computer algebra. 


\section{REFERENCES}

[Altınok 98] Selma Altınok. "Graded Rings Corresponding to Polarised K3 Surfaces and $\mathbb{Q}$-Fano 3-folds." PhD diss., University of Warwick, 1998.

[Altınok 03] Selma Altınok. "Hilbert Series and Applications to Graded Rings." Int. J. Math. Math. Sci. (2003), 397403.

[Altınok et al. 02] Selma Altınok, Gavin Brown, and Miles Reid. "Fano 3-folds, K3 Surfaces and Graded Rings." In Topology and Geometry: Commemorating SISTAG, pp. 2553, Contemp. Math. 314. Providence, RI: AMS, 2002.

[Belcastro 02] Sarah-Marie Belcastro. "Picard Lattices of Families of K3 Surfaces." Comm. Algebra 30:1 (2002) 61-82.

[Bosma and Cannon 04] Wieb Bosma and John Cannon. The Magma Handbook. Available online (magma.maths.usyd. edu.au), 2004.

[Bosma et al. 97] Wieb Bosma, John Cannon, and Catherine Playoust. "The Magma Algebra System. I. The User Language." J. Symbolic Comput. 24:3-4 (1997), 235-265.

[Brown 03] Gavin Brown. "Datagraphs in Algebraic Geometry and K3 Surfaces." In Symbolic and Numerical Scientific Computation (Hagenberg, 2001), pp. 210-224, Lecture Notes in Comput. Sci. 2630. Berlin: Springer, 2003.

[Brown 06] Gavin Brown. "Graded Rings and Special K3 Surfaces." In Discovering Mathematics with Magma, pp.137159, Algorithms and Computational Mathematics 19. Berlin: Springer, 2006.

[Brown and Kerber 05] Gavin Brown and Michael Kerber. "The K3 Surface SQL Database." Available online (http: //www.kent.ac.uk/ims/grdb/), 2005.

[Brown et al. 04] Gavin Brown, Michael Kerber, and Stephen Tawn. "The Graded Ring Database Website." Available online (http://www.kent.ac.uk/ims/grdb/), 2004.

[Buckley 03] Anita Buckley. "Orbifold Riemann-Roch for 3folds and Applications to Calabi-Yaus." PhD diss., University of Warwick, 2003.

[Buckley and Szendrői 05] Anita Buckley and Balázs Szendrői. "Orbifold Riemann-Roch for Threefolds with an Application to Calabi-Yau Geometry." J. Algebraic Geom. 14:4 (2005), 601-622.

[Cannon 05] John Cannon. "The Magma Computational Algebra System." Software available online (magma.maths. usyd.edu.au), 2005.

[Caravantes 05] Jorge Caravantes. "Low Codimension FanoEnriques Threefolds." arXiv:math.AG/0504072, 2005.

[Corti et al. 00] Alessio Corti, Aleksandr Pukhlikov, and Miles Reid. "Fano 3-fold Hypersurfaces." In Explicit Birational Geometry of 3-folds, pp. 175-258, London Math. Soc. Lecture Note Ser. 281. Cambridge: Cambridge Univ. Press, 2000.
[Durfee 79] Alan H. Durfee. "Fifteen Characterizations of Rational Double Points and Simple Critical Points." Enseign. Math. (2) 25:1-2, (1979), 131-163.

[Frantzen 04] Kristina Frantzen. "On K3-Surfaces in Weighted Projective Space." Master's thesis, University of Warwick, 2004.

[Iano-Fletcher 00] A. R. Iano-Fletcher. "Working with Weighted Complete Intersections." In Explicit Birational Geometry of 3-folds, pp. 101-173, London Math. Soc. Lecture Note Ser. 281. Cambridge: Cambridge Univ. Press, 2000 .

[Johnson and Kollár 01] Jennifer M. Johnson and János Kollár. "Fano Hypersurfaces in Weighted Projective 4Spaces." Experiment. Math. 10:1 (2001), 151-158.

[Kasprzyk 05] Alexander Kasprzyk. "Database of Toric Fano 3-folds." Available online (http://www.kent.ac.uk/ims/ $\operatorname{grdb} /), 2005$.

[Kasprzyk 06] Alexander Kasprzyk. "Toric Fano Three-folds with Terminal Singularities." Tohoku Math. J. (2) $58: 1$ (2006), 101-121.

[Kawamata 92] Yujiro Kawamata. "Boundedness of Q-Fano Threefolds." In Proceedings of the International Conference on Algebra, Part 3 (Novosibirsk, 1989), pp. 439-445, Contemp. Math. 131. Providence, RI: AMS, 1992.

[Kreuzer and Skarke 98] Maximilian Kreuzer and Harald Skarke. "Classification of Reflexive Polyhedra in Three Dimensions." Adv. Theor. Math. Phys. 2:4 (1998), 853-871.

[Kreuzer and Skarke 00] Maximilian Kreuzer and Harald Skarke. "Complete Classification of Reflexive Polyhedra in Four Dimensions." Adv. Theor. Math. Phys. 4:6 (2000), 1209-1230.

[Miranda and Persson 89] Rick Miranda and Ulf Persson. "Configurations of $\mathrm{I}_{n}$ Fibers on Elliptic K3 Surfaces." Math. Z. 201:3 (1989), 339-361.

[Papadakis 06] Stavros Argyrios Papadakis. "Type II Unprojection." J. Algebraic Geom. 15:3 (2006), 399-414.

[Papadakis and Reid 04] Stavros Argyrios Papadakis and Miles Reid. "Kustin-Miller Unprojection without Complexes." J. Algebraic Geom. 13:3 (2004), 563-577.

[Reid 80] Miles Reid. "Canonical 3-folds." In Journées de Géometrie Algébrique d'Angers, Juillet 1979/Algebraic Geometry, Angers, 1979, pp. 273-310. Alphen aan den Rijn: Sijthoff \& Noordhoff, 1980.

[Reid 87] Miles Reid. "Young Person's Guide to Canonical Singularities." In Algebraic Geometry, Bowdoin, 1985 (Brunswick, Maine, 1985), pp. 345-414, Proc. Sympos. Pure Math. 46. Providence, RI: AMS, 1987.

[Shimada 00] Ichiro Shimada. "On Elliptic K3 Surfaces." Michigan Math. J. 47:3 (2000), 423-446.

[Shimada and Zhang 01] Ichiro Shimada and De-Qi Zhang. "Classification of Extremal Elliptic K3 Surfaces and Fundamental Groups of Open K3 Surfaces." Nagoya Math. J. 161 (2001), 23-54. 
Gavin Brown, IMSAS, University of Kent, Canterbury, CT2 7NF, UK (gdb@kent.ac.uk)

Received July 10, 2005; accepted August 9, 2005. 